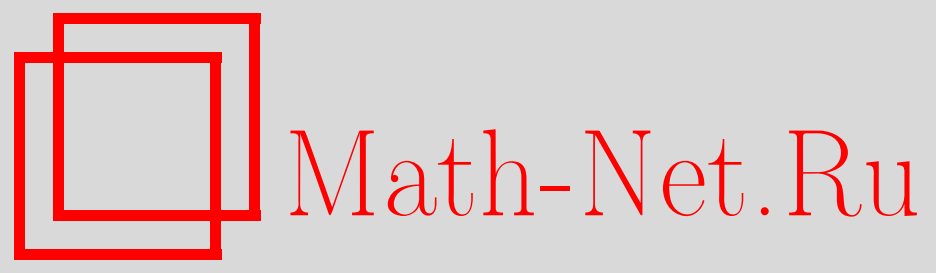

Э. Б. Манукян, Соотношение неопределенностей для квантовых систем с произвольно большим числом частиц и скорость расширения материи в балке, $T M \Phi$, 2014, том 178, номер 2, 290-294

DOI: https://doi.org/10.4213/tmf8527

Использование Общероссийского математического портала Math-Net.Ru подразумевает, что вы прочитали и согласны с пользовательским соглашением http://www . mathnet.ru/rus/agreement

Параметры загрузки:

IP : 3.89 .185 .249

26 апреля 2023 г., $16: 30: 25$

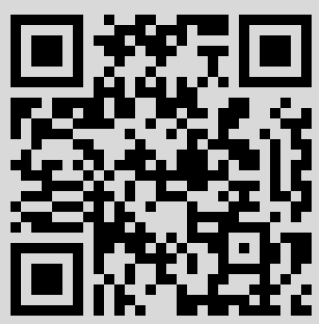




\title{
ФИЗИКА
}

Том 178, № 2

февраль, 2014

(C) 2014 г.

\section{СООТНОШЕНИЕ НЕОПРЕДЕЛЕННОСТЕЙ ДЛЯ КВАНТОВЫХ СИСТЕМ С ПРОИЗВОЛЬНО БОЛЬШИМ ЧИСЛОМ ЧАСТИЦ И СКОРОСТЬ РАСШИРЕНИЯ МАТЕРИИ В БАЛКЕ}

\begin{abstract}
Соотношение неопределенностей для произведения числа частиц $N$ и времени жизни $T$, где последнее рассматривается как мера того, как система сопротивляется изменениям, строго выводится для любой квантовой системы с произвольно большим $N$, для которой нижний край спектра имеет ненулевую ширину и ограничен снизу величиной $N$. Полученное неравенство можно применять при исследовании нетривиальной проблемы скорости расширения материи в балке как функции числа электронов при больших $N$. Решение этой задачи получено на основе наблюдения, что сопротивление увеличению скорости расширения можно определить в рамках квантовой механики в терминах времени жизни при таком увеличении. Показано, что достаточное условие ненулевого времени жизни материи при увеличении скорости расширения состоит в том, что нижний край спектра соответствует нижнему краю энергетической зоны, для которой применение полученного соотношения неопределенностей делается очевидным. Кроме того, показано, что если скорость расширения возрастает с ростом радиуса $R$, то при больших $R$ время жизни убывает не быстрее, чем $1 / R^{3}$. Для полноты и последовательности анализа рассмотрен также формальный предел нулевой ширины. Поскольку полученное соотношение неопределенностей является универсальным, можно ожидать, что оно будет иметь и другие приложения.
\end{abstract}

Ключевые слова: соотношение неопределенностей, квантовые системы с произвольно большим числом частиц, скорость расширения материи в балке, время жизни при увеличении скорости расширения.

DOI: $10.4213 / \operatorname{tmf} 8527$

Для любой квантовой системы с произвольно большим числом частиц $N$, нижний край спектра которой ограничен снизу величиной $N$ и соответствует нижнему краю энергетической зоны, мы строго выводим соотношение неопределенностей для произведения $N$ и времени жизни системы $T$, которое рассматривается в качестве меры того, как система сопротивляется всяким изменениям своего начального состояния.

${ }^{*}$ The Institute for Fundamental Study, Naresuan University, Phitsanulok, Thailand. E-mail: manoukian_eb@hotmail.com 
Соответствующее неравенство далее применяется для исследования ключевой проблемы скорости расширения материи. Фундаментальное свойство материи состоит в том, что она расширяется как функция $N$ и увеличивается в размере, и этот факт можно строго выразить количественно с помощью утверждения, что для ненулевой вероятности наличия электронов внутри сферы радиуса $R$ при больших $N$ [1] этот радиус растет не медленнее, чем $N^{1 / 3}$. Это нетрудно видеть из явного неравенства, приведенного ниже. Таким образом, не удивительно, что материя занимает такой большой объем. Здесь стоит вспомнить знаменитое историческое высказывание Эренфеста [2], [3] о материи, с которым он обратился к Паули в 1931 г. по случаю присуждения ему медали Лоренца за открытие этого эффекта: “Возъмем кусок металла или камешек. При размышлении об этом нас изумляет, что такое количество материи вынуждено занимать такой большой обгем". После чего он заявил, что причиной этого является принцип Паули: "Oтвет: только благодаря принципу Паули - два электрона не могут находитъся в одном состоянии".

В равной мере интересно исследовать скорость расширения материи и выяснить, замедляется ли оно или протекает быстрее. Косвенный способ исследовать это состоит в том, чтобы изучить возможное сопротивление материи при любых изменениях скорости расширения, а затем посмотреть, убывает или возрастает это сопротивление при расширении. В квантовой физике сопротивление изменению состояния можно определить в терминах времени жизни $T$. Имея это в виду, покажем, что достаточное условие ненулевого времени жизни материи при увеличении скорости расширения состоит в том, чтобы нижний край спектра гамильтониана рассматриваемой системы являлся нижним краем ненулевой энергетической зоны, а затем установим, в частности, что если скорость расширения увеличивается с ростом $R$ при больших $R$, то время жизни с необходимостью убывает не быстрее чем $1 / R^{3}$.

Мы применяем полученное соотношение неопределенностей к весьма нетривиальной квантовой системе с гамильтонианом материи в балке с $N$ электронами вида

$$
H=\sum_{i=1}^{N} \frac{\vec{p}_{i}^{2}}{2 m}+\sum_{i<j}^{N} \frac{e^{2}}{\left|\vec{x}_{i}-\vec{x}_{j}\right|}-\sum_{i=1}^{N} \sum_{j=1}^{k} \frac{Z_{j} e^{2}}{\left|\vec{x}_{i}-\vec{R}_{j}\right|}+\sum_{i<j}^{k} \frac{Z_{i} Z_{j} e^{2}}{\left|\vec{R}_{i}-\vec{R}_{j}\right|}
$$

где $m$ - масса электрона, а $\vec{x}_{i}$ и $\vec{R}_{j}$ - координаты электронов и ядер соответственно. Рассмотрим также нейтральную материю, для которой $\sum_{j=1}^{k} Z_{j}=N$. Несомненно, одной из наиболее важных и серьезных проблем, решенных квантовой физикой, является проблема устойчивости материи. Принцип Паули оказывается не только достаточным, но и необходимым для устойчивости. Ранние исследования устойчивости материи восходят к классическим работам Дайсона и Ленарда [3], [4], а более недавние - к работам Лейба и Тиринга [5], [6]. Фундаментальное свойство материи состоит в том, что нижний край спектра $E_{N} \sim N$, соответствующий приведенному выше гамильтониану, содержит $N$ в единственной степени, в отличие от материи, на которую не налагается принципа Паули, - так называемой "бозонной материи", для которой $E_{N} \sim N^{\alpha}$, где $\alpha>1$ [3], [7]-[12]. Из такого степенного поведения при $\alpha>1$, в отличие от обыкновенной материи, следует неустойчивость ${ }^{1)}$, которая заключается в том, что образование одной системы, состоящей из $2 N+2 N$ частиц, является

1) Педагогическая трактовка проблемы устойчивости материи приведена, например, в работе [12]. 
предпочтительным по сравнению с образованием двух отдельных систем, сведенных вместе, каждая из которых состоит из $N+N$ частиц, а энергия, выделяемая при слиянии двух систем в одну, пропорциональная $(2 N)^{\alpha}-2 N^{\alpha}$, будет подавляюще большой при больших $N$, например при $N \sim 10^{23}$.

Элементарное свойство, выведенное подробно в работе [1], которое следует из гамильтониана (1) и которое легко интерпретируется физически, состоит в том, что вероятность нахождения $N$ электронов в пределах радиуса $R, \operatorname{Prob}\left[\left|\vec{x}_{1}\right| \leqslant R, \ldots\right.$, $\left.\left|\vec{x}_{N}\right| \leqslant R\right]$, удовлетворяет простому неравенству:

$$
\operatorname{Prob}\left[\left|\overrightarrow{x_{1}}\right| \leqslant R, \ldots,\left|\overrightarrow{x_{N}}\right| \leqslant R\right]\left(\frac{N}{v_{R}}\right)^{2 / 5}<\left(\frac{1}{a_{0}^{3}}\right)^{2 / 5} 1.846\left[1+Z^{2 / 3}\right]^{6 / 5},
$$

где $a_{0}=1 / m e^{2}, v_{R}=4 \pi R^{3} / 3$, a $Z=\max _{j} Z_{j}$, причем $|e| Z_{j}$ - заряд $j$-го ядра в материи $(\hbar=1)$. Отметим здесь, что при ненулевой вероятности нахождения электронов внутри сферы радиуса $R$ соответствующий объем $v_{R}$ увеличивается при $N \rightarrow \infty$ никак не медленнее, чем первая степень $N$, поскольку в противном случае левая часть неравенства (2) стремилась бы к бесконечности, что противоречит наличию конечной верхней границы правой части.

Чтобы вывести обсуждаемое соотношение неопределенностей, обозначим через $-c_{N} N$ нижний край спектра, где $c_{N}<c-$ конечная положительная константа, при всех больших $N$. Покажем, что достаточное условие ненулевого времени жизни материи, т. е. ее сопротивления изменению скорости расширения с ростом $N$, заключается в том, что нижний край спектра является нижним краем энергетической зоны $\left(-c_{N},\left[-c_{N}+\Delta_{N}\right]\right) N$, где $\Delta_{N} \leqslant \Delta$ при больших $N$. Соответственно, можно рассмотреть масштабированный гамильтониан $H / N$ и описать нижний край спектра посредством состояния следующим образом:

$$
|\psi\rangle=\int_{0}^{\infty} d \rho \chi_{\Delta_{N}}(\rho) \delta\left(\left[H / N+c_{N}\right]-\rho\right)|\psi\rangle
$$

где $\chi_{\Delta_{N}}(\rho)$ равно единице при $\rho$, лежащем в интервале $\left(0, \Delta_{N}\right)$, и равно нулю в других случаях. Умножая это уравнение на $\langle\psi|$ и используя нормируемость состояния $|\psi\rangle$, получаем

$$
\begin{aligned}
\langle\psi \mid \psi\rangle & =\int_{0}^{\infty} \frac{d \rho}{2 \pi} \chi_{\Delta_{N}}(\rho) F(\rho)=1, \\
F(\rho) & =2 \pi\left\langle\psi\left|\delta\left(\left[H / N+c_{N}\right]-\rho\right)\right| \psi\right\rangle .
\end{aligned}
$$

Состояние $|\psi\rangle$ развивается во времени следующим образом:

$$
\begin{aligned}
\left|\psi_{t}\right\rangle & =e^{-i H t}|\psi\rangle=\int_{0}^{\infty} d \rho \chi_{\Delta_{N}}(\rho) e^{-i\left(-c_{N}+\rho\right) N t} \delta\left(\left[H / N+c_{N}\right]-\rho\right)|\psi\rangle, \\
\left\langle\psi \mid \psi_{t}\right\rangle & =\int_{0}^{\infty} \frac{d \rho}{2 \pi} e^{-i\left(-c_{N}+\rho\right) N t} \chi_{\Delta_{N}}(\rho) F_{N}(\rho),
\end{aligned}
$$

где $\left|\left\langle\psi \mid \psi_{t}\right\rangle\right|^{2}$ - вероятность того, что система в момент времени $t$ находится в своем начальном состоянии, и эта величина называется вероятностъю выживания. Время жизни определяется как

$$
T=\frac{1}{2} \int_{-\infty}^{\infty} d t\left|\left\langle\psi \mid \psi_{t}\right\rangle\right|^{2}=\frac{1}{2 N} \int_{0}^{\infty} \frac{d \rho}{2 \pi} \chi_{\Delta_{N}}(\rho)\left|F_{N}(\rho)\right|^{2} .
$$


С другой стороны, используя неравенство Шварца, из (4) получаем

$$
1=\left|\int_{0}^{\infty} \frac{d \rho}{2 \pi} \chi_{\Delta_{N}}(\rho) F(\rho)\right|^{2} \leqslant\left(\int_{0}^{\infty} \frac{d \rho}{2 \pi} \chi_{\Delta_{N}}(\rho)\right)\left(\int_{0}^{\infty} \frac{d \rho}{2 \pi} \chi_{\Delta_{N}}(\rho)\left|F_{N}(\rho)\right|^{2}\right) .
$$

Тогда из (8) получаем основное неравенство:

$$
1 \leqslant \frac{1}{\pi} N T \Delta_{N} \leqslant \frac{1}{\pi} N T \Delta
$$

или

$$
\frac{\pi}{T \Delta} \leqslant N
$$

Выражая $N$ в терминах $T$ в левой части (2), из (11) получаем

$$
\operatorname{Prob}\left[\left|\overrightarrow{x_{1}}\right| \leqslant R, \ldots,\left|\overrightarrow{x_{N}}\right| \leqslant R\right]\left(\frac{1}{v_{R} T}\right)^{2 / 5}<\left(\frac{\Delta}{\pi a_{0}^{3}}\right)^{2 / 5} 1.846\left[1+Z^{2 / 3}\right]^{6 / 5},
$$

где левая часть представляет собой нижнюю границу левой части (2) с точностью до члена $(\Delta / \pi)^{2 / 5}$, появляющегося в правой части неравенства $(12)$.

Эта формула представляет интерес с различных точек зрения. Неравенство не запрещает постоянства или увеличения времени жизни $T$ с ростом $R$ при больших $R$, т. е. постоянства или уменьшения скорости расширения материи. Это показывает, однако, что если скорость расширения увеличивается с ростом $R$, то при больших $R$ время жизни убывает не быстрее чем $1 / R^{3}$, при $R \rightarrow \infty$, поскольку в противном случае левая часть неравенства (12) стремилась бы к бесконечности, что противоречит тому, что его правая часть при ненулевой вероятности имеет конечную верхнюю границу. Наконец, из соотношений (10)-(12) мы также выводим ожидаемый квантово-механический результат в формальном пределе нулевой ширины $\Delta_{N} \equiv \Delta \rightarrow 0$, поскольку согласно этим соотношениям мы должны одновременно получить $T \rightarrow \infty$, что соответствует изолированному связанному состоянию, без изменений в соответствующем состоянии (с точностью до фазового множителя), что согласуется с нашим анализом.

Поскольку полученное соотношение неопределенностей является универсальным, можно ожидать, что оно применимо и к другим задачам. Такие дополнительные применения, а также трактовки "бозонной материи" требуют некоторых специальных средств и будут рассмотрены в отдельной работе.

Благодарности. Автор благодарит своих коллег из Institute for Fundamental Study за интерес, который они проявили к настоящей работе.

\section{Список литературы}

[1] E. B. Manoukian, S. Sirininlakul, Phys. Rev. Lett., 95:19 (2005), 190402, 3 pp.

[2] P. Ehrenfest, "Ansprache zur Verleihung der Lorentz-Medaille an Professor Wolfgang Pauli am 31 Oktober 1931", Paul Ehrenfest: Collected Scientific Papers, ed. M. J. Klein, North-Holland, Amsterdam, 1959, 617.

[3] F. J. Dyson, J. Math. Phys., 8:8 (1967), 1538-1545.

[4] J. F. Dyson, A. Lenard, J. Math. Phys., 8:3 (1967), 423-434.

[5] A. Lenard, F. J. Dyson, J. Math. Phys., 9:5 (1968), 698-711. 
[6] E. Lieb, W. E. Thirring, Phys. Rev. Lett., 35:11 (1975), 687-689; 35:16 (1975), 1116-1116.

[7] E.H. Lieb, The Stability of Matter: From Atoms to Stars., Selecta of E.H. Lieb, ed. W. Thirring, Springer, New York, 1991.

[8] E. B. Manoukian, C. Muthaporn, Progr. Theor. Phys., 110:2 (2003), 385-391.

[9] C. Muthaporn, E. B. Manoukian, Phys. Lett. A, 321:3 (2004), 152-154.

[10] E. B. Manoukian, S. Sirininlakul, Phys. Lett. A, 332:1-2 (2004), 54-59.

[11] E. B. Manoukian, C. Muthaporn, S. Sirininlakul, Phys. Lett. A, 352:6 (2006), 488-490.

[12] E. B. Manoukian, Quantum Theory: A Wide Spectrum, Springer, Amsterdam, 2006.

Поступила в редакцию 8.03.2013,

после доработки 31.05.2013 